\title{
A regularity theory for scalar local minimizers of splitting-type variational integrals
}

\author{
Michael Bildhauer, Martin Fuchs And Xiao Zhong
}

\begin{abstract}
Starting from Giaquinta's counterexample [12] we introduce the class of splitting functionals being of $(p, q)$-growth with exponents $p \leq q<\infty$ and show for the scalar case that locally bounded local minimizers are of class $C^{1, \mu}$. Note that to our knowledge the only $C^{1, \mu}$-results without imposing a relation between $p$ and $q$ concern the case of two independent variables as it is outlined in Marcellini's paper [15], Theorem A, and later on in the work of Fusco and Sbordone [10], Theorem 4.2.
\end{abstract}

Mathematics Subject Classification (2000): 49N60.

\section{Introduction}

In 1987 Giaquinta [12] showed that the function

$$
\begin{aligned}
u_{0}(x) & :=\sqrt{\frac{n-4}{24}} x_{n}^{2} / \sqrt{x_{1}^{2}+\ldots+x_{n-1}^{2}}, \\
x \in B_{1}(0) & =\left\{y \in \mathbb{R}^{n}:|y|<1\right\},
\end{aligned}
$$

is a local minimizer of the energy

$$
J[w]=\int_{B_{1}(0)}\left[\sum_{i=1}^{n-1}\left(\partial_{i} w\right)^{2}+\frac{1}{2}\left(\partial_{n} w\right)^{4}\right] \mathrm{d} x,
$$

provided that $n \geq 6$. Since $u_{0}$ is unbounded, Giaquinta's example clearly demonstrates that for anisotropic variational integrals in general no regularity results for local minimizers can be expected, and this even concerns the scalar situation! So one may ask for an admissible range of anisotropy implying that local minimizers are locally bounded (and even share a higher degree of regularity) or one may try to compensate the anisotropic structure of the integrand by adding certain natural

Received March 16, 2007; accepted in revised form May 23, 2007. 
hypotheses on the behaviour of the local minimizer leading to its regularity. But let us first have a closer look at Giaquinta's energy $J$. If we write $z=\left(\tilde{z}, z_{n}\right)$, $\tilde{z}:=\left(z_{1}, \ldots, z_{n-1}\right)$, for elements $z$ of $\mathbb{R}^{n}, n \geq 2$, then the energy density occurring in (1.1) is of splitting form, i.e. we have an integrand $F: \mathbb{R}^{n} \rightarrow[0, \infty)$ such that

$$
F(z)=f(\tilde{z})+g\left(z_{n}\right)
$$

with $C^{2}$-functions $f: \mathbb{R}^{n-1} \rightarrow[0, \infty), g: \mathbb{R} \rightarrow[0, \infty)$ satisfying with exponents $1<p \leq q<\infty$ and with constants $\lambda, \Lambda>0$ the ellipticity conditions $\left(y, z \in \mathbb{R}^{n}\right)$

$$
\begin{aligned}
\lambda\left(1+|\tilde{z}|^{2}\right)^{\frac{p-2}{2}}|\tilde{y}|^{2} & \leq D^{2} f(\tilde{z})(\tilde{y}, \tilde{y}) \leq \Lambda\left(1+|\tilde{z}|^{2}\right)^{\frac{p-2}{2}}|\tilde{y}|^{2}, \\
\lambda\left(1+\left|z_{n}\right|^{2}\right)^{\frac{q-2}{2}}\left|y_{n}\right|^{2} & \leq D^{2} g\left(z_{n}\right)\left(y_{n}, y_{n}\right) \leq \Lambda\left(1+\left|z_{n}\right|^{2}\right)^{\frac{q-2}{2}}\left|y_{n}\right|^{2} .
\end{aligned}
$$

Of course (1.4) could be stated in a simpler form but (1.2) just serves as a model case: in fact we could consider any decomposition $z=\left(z^{(1)}, z^{(2)}\right)$ of the vector $z$ and replace (1.2) by $F(z)=f\left(z^{(1)}\right)+g\left(z^{(2)}\right)$ with $f$ and $g$ satisfying the appropriate versions of (1.3) and (1.4). We also like to remark that for $p \geq 2$ the degenerate variants of (1.3), (1.4) can be considered so that Giaquinta's energy (1.1) is included. Now, if $\Omega$ is an open set in $\mathbb{R}^{n}$, we let

$$
I[w, \Omega]=\int_{\Omega} F(\nabla w) \mathrm{d} x,
$$

and since (1.3), (1.4) imply the growth estimate

$$
a|z|^{p}-b \leq F(z) \leq A|z|^{q}+B, z \in \mathbb{R}^{n},
$$

with constants $a, A>0, b, B \geq 0$, it is natural to call a function $u$ from the local Sobolev space $W_{p, \text { loc }}^{1}(\Omega)$ (see [1] for a definition) a local minimizer of $I$ from (1.5) iff $I\left[u, \Omega^{\prime}\right]<\infty$ and $I\left[u, \Omega^{\prime}\right] \leq I\left[w, \Omega^{\prime}\right]$ for any open set $\Omega^{\prime}$ s.t. $\Omega^{\prime} \Subset \Omega$ and for all $w \in W_{p, \text { loc }}^{1}(\Omega)$ such that $\operatorname{spt}(u-w) \Subset \Omega^{\prime}$.

Coming back to the regularity problem for local minimizers $u$, it turns out that conditions of the form

$$
q \leq c(n) p
$$

are sufficient for the local boundedness of the function $u$ and also for its higher regularity. Here $c(n)$ is a constant depending on $n$ giving rather large values if $n$ is small but with the unpleasant property $c(n) \rightarrow 1$ as $n \rightarrow \infty$. We mention the contributions of Fusco and Sbordone [10], Marcellini [15, 16] and Hong [14], where one also finds further references. It should be remarked that results of this type usually do not refer to a splitting structure of the integrand $F$ as stated in (1.2): in place of this one works with (1.6) combined with an appropriate ellipticity condition, or one just requires that

$$
\lambda\left(1+|z|^{2}\right)^{\frac{p-2}{2}}|y|^{2} \leq D^{2} F(z)(y, y) \leq \Lambda\left(1+|z|^{2}\right)^{\frac{q-2}{2}}|y|^{2},
$$


which obviously implies the validity of (1.6). The reader should note that for integrands $F$ satisfying (1.2) the conditions (1.3), (1.4) do not imply (1.8), and that conversely (1.3), (1.4) can not be deduced from (1.8).

Let us now look at a special situation for which it is possible to improve (1.7): if $\Omega$ is a bounded domain and if $u$ is an $I[\cdot, \Omega]$-minimizer for boundary data $\bar{u} \in$ $L^{\infty}(\Omega)$, which means that we are given $\bar{u} \in W_{p}^{1}(\Omega) \cap L^{\infty}(\Omega)$ such that $I[\bar{u}, \Omega]<$ $\infty$ and where the boundary condition has to be understood in the sense that $u-$ $\bar{u} \in \stackrel{\circ}{W}_{p}^{1}(\Omega)$, then the maximum-principle implies $u \in L^{\infty}(\Omega)$. From this point of view it makes sense to study local minimizers from the space $L_{\text {loc }}^{\infty}(\Omega)$. Assuming this together with (1.8), Choe [7] proved the smoothness of $u$ under the dimensionless condition $q<p+1$, which was replaced by $q<p+2$ in [2] and [3]. We wish to remark that the bound $q<p+2$ was first introduced by Esposito, Leonetti and Mingione in the paper [8]. If the integrand $F$ is of splitting type satisfying (1.3), (1.4) with $q \leq 2 p$, then in the recent paper [6] we could show the differentiability of local minimizers $u \in L_{\text {loc }}^{\infty}(\Omega)$ which leads to a partial improvement of earlier results of Ural'tseva and Urdaletova [19]. In the present note we are going to apply new methods leading to the following results:

Theorem 1.1. Let $1<p \leq q<\infty$, and let $F$ satisfy (1.2), (1.3) and (1.4). Consider a local $I[\cdot, \Omega]$-minimizer u of class $W_{p, \mathrm{loc}}^{1}(\Omega) \cap L_{\mathrm{loc}}^{\infty}(\Omega)$. Then we have:

i) $\nabla u \in L_{\text {loc }}^{m}\left(\Omega ; \mathbb{R}^{n}\right)$ for any finite exponent $m$.

ii) If in addition $p \geq 2$, then $u \in C^{1, \mu}(\Omega)$ holds for all $\mu<1$.

Remark 1.2. If the case of degenerate ellipticity is considered in (1.3) and (1.4), i.e. if the 1 in these inequalities is replaced by 0 , then we have Theorem 1.1, i) under the restriction that $p \geq 2$. We like to mention that for the non-splitting but degenerate case higher integrability of $\nabla u$ has been established earlier in [9] working with the bound $q<p(n+1) / n$, we also refer to [5].

Remark 1.3. We want to emphasize again that our results are not limited to the specific decomposition (1.2). With minor modifications we can also discuss the integrand

$$
F(z)=\sum_{i=1}^{n}\left(1+z_{i}^{2}\right)^{p_{i} / 2}, \quad z \in \mathbb{R}^{n},
$$

with exponents $1<p_{i}<\infty$. Alternatively we may consider a decomposition

$$
F(z):=F^{(1)}\left(z^{(1)}\right)+F^{(2)}\left(z^{(2)}\right),
$$

where for example $z^{(1)}:=\left(z_{1}, \ldots, z_{k}\right), z^{(2)}:=\left(z_{k+1}, \ldots z_{n}\right)$ with $1 \leq k<n$ and where $F^{(1)}$ and $F^{(2)}$ satisfy ellipticity conditions like (1.3) and (1.4) with exponents $p_{1}$ and $p_{2}$. Another possible extension concerns the decomposition $F(z)=f(z)+g\left(z_{n}\right)$, where now $f$ depends on the full gradient. In addition we can include the dependence of $g$ on more than one partial derivative provided we have the appropriate variants of (1.3) and (1.4). 
Remark 1.4. Going through the proof of Theorem 1.1 one easily checks that the explicit additive structure of $F$ itself formulated in (1.2) is not really needed. In fact, if we drop (1.2) and replace (1.3) and (1.4) by the assumption

$$
\begin{aligned}
\lambda\left[\left(1+|\tilde{z}|^{2}\right)^{\frac{p-2}{2}}|\tilde{y}|^{2}+\left(1+\left|z_{n}\right|^{2}\right)^{\frac{q-2}{2}}\left|y_{n}\right|^{2}\right] & \leq D^{2} F(z)(y, y) \\
& \leq \Lambda\left[\left(1+|\tilde{z}|^{2}\right)^{\frac{p-2}{2}}|\tilde{y}|^{2}+\left(1+\left|z_{n}\right|^{2}\right)^{\frac{q-2}{2}}\left|y_{n}\right|^{2}\right],
\end{aligned}
$$

then we still have our results stated above.

Remark 1.5. It is easy to extend Theorem 1.1 to non-autonomous energies $F(x, z)$, $x \in \bar{\Omega}, z \in \mathbb{R}^{n}$, provided $D_{x} D_{z} F(x, z)$ satisfies a natural growth condition.

Remark 1.6. In the case of vector-valued functions we have much weaker results which are summarized in [4]. However, if $n=2$, then Theorem 1.1, ii) is true.

As an application of Theorem 1.1 we consider the following variant of Giaquinta's energy which was introduced by Fusco and Sbordone (see [10], formula (3.4)): for $q>2$ and a positive constant $c$ let

$$
J_{q}[w]:=\int_{B_{1}(0)}\left[\sum_{i=1}^{n-1}\left(\partial_{i} w\right)^{2}+\frac{2 c}{q}\left|\partial_{n} u\right|^{q}\right] \mathrm{d} x .
$$

If $n \leq 3$, then according to Theorem 3.1 in [10] any local $J_{q}$-minimizer is locally bounded independent of the value of $q$, whereas Fusco and Sbordone obtain $C^{1, \alpha}$-regularity for the non-degenerate variant of $J_{q}$ if $q \leq 2 n /(n-2)$ is satisfied (see Theorem 4.2 in [10]), i.e. they require the bound $q \leq 6$ in the 3D-case. But Theorem 1.1 combined with the local boundedness result of [10] shows

Corollary 1.7. If $n \leq 3$ and if $q \geq 2$ is arbitrary, then all local minima of the functional $J_{q}$ belong to the class $C^{1, \alpha}\left(B_{1}(0)\right)$ for an exponent $\alpha>0$.

We leave it to the reader to give a version of this corollary valid for more general functionals on three-dimensional domains.

Finally, we look at the global minimization problem for which we can state

Corollary 1.8. Under the hypotheses of Theorem 1.1 or of Corollary 1.7 consider a function $\bar{u} \in L^{\infty}(\Omega)$ with finite energy. Then, if $u$ denotes the unique minimizing map for boundary values $\bar{u}$, we have smoothness of $u$ in the interior of $\Omega$.

ACKNOWLEDGement. A part of this paper was finished during the stay of M. Bildhauer and M. Fuchs at the University of Jyväskylä in November 2006. We like to thank the members of the Dept. of Math. for their hospitality. 


\section{Proof of Theorem 1.1}

Obviously Theorem 1.1, ii) follows from part i) combined with Lemma 3.1. To prove i) we fix a ball $B:=B_{R}\left(x_{0}\right)$ with compact closure in $\Omega$ and consider the mollification $(u)_{\epsilon}$ with a small radius $\epsilon>0$. Let $u_{\epsilon}$ denote the unique Lipschitz function minimizing $I[\cdot, B]$ in the class of all Lipschitz mappings $\bar{B} \rightarrow \mathbb{R}$ for boundary values $(u)_{\epsilon}$, i.e. $u_{\epsilon}$ denotes the Hilbert-Haar solution (see, e.g., [17], Theorem 4, page 162). The main properties of this approximation are summarized in

Lemma 2.1. i) Passing to the limit $\epsilon \rightarrow 0$ we have

$$
u_{\epsilon} \rightarrow u \quad \text { in } W_{p}^{1}(B), \quad \int_{B} F\left(\nabla u_{\epsilon}\right) \mathrm{d} x \rightarrow \int_{B} F(\nabla u) \mathrm{d} x .
$$

ii) $\left\|u_{\epsilon}\right\|_{L^{\infty}(B)}$ is bounded independent of $\epsilon$.

Proof. i) The minimality of $u_{\epsilon}$ implies

$$
\int_{B} F\left(\nabla u_{\epsilon}\right) \mathrm{d} x \leq \int_{B} F\left(\nabla(u)_{\epsilon}\right) \mathrm{d} x,
$$

and from Jensen's inequality we deduce

$$
\int_{B} F\left(\nabla(u)_{\epsilon}\right) \mathrm{d} x \leq \int_{B} F(\nabla u) \mathrm{d} x+O(\epsilon)
$$

with $O(\epsilon) \rightarrow 0$ as $\epsilon \rightarrow 0$. Due to the growth of $F$ we find $\sup _{\epsilon>0}\left\|u_{\epsilon}\right\|_{W_{p}^{1}(B)}<$ $\infty$, so that $u_{\epsilon} \rightarrow \bar{u}$ in $W_{p}^{1}(B)$ for some function $\bar{u}$ from this class. By lower semicontinuity it holds

$$
\int_{B} F(\nabla \bar{u}) \mathrm{d} x \leq \liminf _{\epsilon \rightarrow 0} \int_{B} F\left(\nabla u_{\epsilon}\right) \mathrm{d} x,
$$

hence

$$
\int_{B} F(\nabla \bar{u}) \mathrm{d} x \leq \int_{B} F(\nabla u) \mathrm{d} x
$$

On the other hand we have $u_{\epsilon}-(u)_{\epsilon} \in \stackrel{\circ}{W}_{p}^{1}(B)$, which means $\bar{u}-u \stackrel{\circ}{W_{p}}{ }^{1}(B)$. Clearly $u$ minimizes $I[\cdot, B]$ w.r.t. its own boundary values, and the strict convexity of $F$ implies $\bar{u}=u$. thus

ii) Let $v:=\min \left\{u_{\epsilon}, \sup _{\partial B}(u)_{\epsilon}\right\}$. Then $v$ is Lipschitz on $B$ with trace $(u)_{\epsilon}$,

$$
I\left[u_{\epsilon}, B\right] \leq I[v, B]
$$

and in conclusion $u_{\epsilon} \leq \sup _{\partial B}(u)_{\epsilon}$. In the same way we obtain $u_{\epsilon} \geq \min _{\partial B}(u)_{\epsilon}$, and since $u \in L^{\infty}(B)$, the claim follows from these estimates. 
Lemma 2.2. The functions $u_{\epsilon}$ are of class $C^{1, \mu}(B) \cap W_{2, \text { loc }}^{2}(B)$ for any $\mu<1$.

Proof. This result is standard and can be found in the textbook of Massari and Miranda, [17], Theorem 5, page 166. The first lines of the proof of Lemma 3.1 summarize the idea observing that by definition of $u_{\epsilon}$ we here already have $\nabla u_{\epsilon} \epsilon$ $L^{\infty}\left(B ; \mathbb{R}^{n}\right)$.

Lemma 2.3 (Variants of Caccioppoli's inequality). For any numbers $\alpha, \beta \geq 0$ and for all $\eta \in C_{0}^{\infty}(B)$ s.t. $0 \leq \eta \leq 1$ we have

$$
\begin{aligned}
& \int_{B} D^{2} F\left(\nabla u_{\epsilon}\right)\left(\partial_{n} \nabla u_{\epsilon}, \partial_{n} \nabla u_{\epsilon}\right) \Gamma_{n, \epsilon}^{\frac{\alpha}{2}} \eta^{2} \mathrm{~d} x \\
& \leq c(\alpha) \int_{\Omega} D^{2} F\left(\nabla u_{\epsilon}\right)(\nabla \eta, \nabla \eta) \Gamma_{n, \epsilon}^{\frac{\alpha+2}{2}} \mathrm{~d} x, \\
& \int_{B} D^{2} F\left(\nabla u_{\epsilon}\right)\left(\partial_{\gamma} \nabla u_{\epsilon}, \partial_{\gamma} \nabla u_{\epsilon}\right) \tilde{\Gamma}_{\epsilon}^{\frac{\beta}{2}} \eta^{2} \mathrm{~d} x \\
& \quad \leq c(\beta) \int_{B} D^{2} F\left(\nabla u_{\epsilon}\right)(\nabla \eta, \nabla \eta) \tilde{\Gamma}_{\epsilon}^{\frac{\beta+2}{2}} \mathrm{~d} x .
\end{aligned}
$$

In (2.2) (and in what follows) we always take the sum w.r.t. $\gamma$ from 1 to $n-1$. $c(\alpha), c(\beta)$ denote positive constants independent of $\varepsilon$, and we have set: $\Gamma_{n, \epsilon}=$ $1+\left(\partial_{n} u_{\epsilon}\right)^{2}, \tilde{\Gamma}_{\epsilon}=1+\left|\tilde{\nabla} u_{\epsilon}\right|^{2}, \tilde{\nabla}:=\left(\partial_{1}, \ldots, \partial_{n-1}\right)$.

Proof. See, e.g., the beginnings of Section 3 and Section 4 of [6]. We like to remark that by Lemma 2.2 any first partial derivative of $u_{\epsilon}$ is a solution of an uniformly elliptic equation with continuous coefficients so that by standard potential theory we get $\partial_{k} u_{\epsilon} \in W_{m, \text { loc }}^{1}(B)$ for any $m<\infty$ and all $k=1, \ldots, n$, i.e. $u_{\epsilon} \in W_{m, \text { loc }}^{2}(B)$ for all $m<\infty$. For this reason the calculations leading to (2.1) and (2.2), which were carried out in [6] for a different type of approximation, can be justified in the present setting.

Now, with $\alpha \geq 0$ and $\eta \in C_{0}^{\infty}(B), 0 \leq \eta \leq 1$, being fixed for the moment we consider the expression

$$
\int_{B} \eta^{2} \Gamma_{n, \epsilon}^{\frac{q+2+\alpha}{2}} \mathrm{~d} x=\int_{B} \eta^{2} \Gamma_{n, \epsilon}^{\frac{q+\alpha}{2}} \mathrm{~d} x+\int_{B} \partial_{n} u_{\epsilon} \partial_{n} u_{\epsilon} \eta^{2} \Gamma_{n, \epsilon}^{\frac{q+\alpha}{2}} \mathrm{~d} x
$$

which is well defined by the Lipschitz continuity of $u_{\epsilon}$, and according to Lemma 2.2 we are allowed to integrate by parts in the second term on the r.h.s. of (2.3), i.e.

$$
\int_{B} \partial_{n} u_{\epsilon} \partial_{n} u_{\epsilon} \eta^{2} \Gamma_{n, \epsilon}^{\frac{q+\alpha}{2}} \mathrm{~d} x=-\int_{B} u_{\epsilon} \partial_{n}\left[\partial_{n} u_{\epsilon} \eta^{2} \Gamma_{n, \epsilon}^{\frac{q+\alpha}{2}}\right] \mathrm{d} x
$$


Using Lemma 2.1 ii), we see that

$$
\begin{aligned}
& \int_{B} \eta^{2} \Gamma_{n, \epsilon}^{\frac{q+\alpha+2}{2}} \mathrm{~d} x \\
& \leq c\left[\int_{B} \eta^{2} \Gamma_{n, \epsilon}^{\frac{q+\alpha}{2}} \mathrm{~d} x+\int_{B} \eta|\nabla \eta|\left|\partial_{n} u_{\epsilon}\right| \Gamma_{n, \epsilon}^{\frac{q+\alpha}{2}} \mathrm{~d} x+\int_{B} \eta^{2}\left|\partial_{n} \partial_{n} u_{\epsilon}\right| \Gamma_{n, \epsilon}^{\frac{q+\alpha}{2}} \mathrm{~d} x\right] \\
& =: c\left[\int_{B} \eta^{2} \Gamma_{n, \epsilon}^{\frac{q+\alpha}{2}}+I_{1}+I_{2}\right]
\end{aligned}
$$

with a constant $c$ depending also on $\alpha$ but being independent of $\varepsilon$ and $\eta$. In order to handle $I_{1}$ and $I_{2}$ we will apply Young's inequality with small parameter $\tau$ which enables us to absorb terms in the 1.h.s. of (2.4). We have

$$
\begin{aligned}
I_{1} & \leq \int_{B} \eta|\nabla \eta| \Gamma_{n, \epsilon}^{\frac{q+1+\alpha}{2}} \mathrm{~d} x \leq \tau \int_{B} \eta^{2} \Gamma_{n, \epsilon}^{\frac{q+2+\alpha}{2}} \mathrm{~d} x+c(\tau) \int_{B}|\nabla \eta|^{2} \Gamma_{n, \epsilon}^{\frac{q+\alpha}{2}} \mathrm{~d} x, \\
I_{2} & =\int_{B} \eta^{2}\left|\partial_{n} \partial_{n} u_{\epsilon}\right| \Gamma_{n, \epsilon}^{\frac{q-2+\alpha}{4}} \Gamma_{n, \epsilon}^{\frac{q+2+\alpha}{4}} \mathrm{~d} x \\
& \leq \tau \int_{B} \Gamma_{n, \epsilon}^{\frac{q+2+\alpha}{2}} \eta^{2} \mathrm{~d} x+c(\tau) \int_{B} \eta^{2}\left|\partial_{n} \partial_{n} u_{\epsilon}\right|^{2} \Gamma_{n, \epsilon}^{\frac{q-2+\alpha}{2}} \mathrm{~d} x,
\end{aligned}
$$

which implies by (2.4)

$$
\begin{gathered}
\int_{B} \eta^{2} \Gamma_{n, \epsilon}^{\frac{q+2+\alpha}{2}} \mathrm{~d} x \leq c\left[\int_{B}\left(\eta^{2}+|\nabla \eta|^{2}\right) \Gamma_{n, \epsilon}^{\frac{q+\alpha}{2}} \mathrm{~d} x+J\right], \\
J:=\int_{B} \eta^{2}\left|\partial_{n} \partial_{n} u_{\epsilon}\right|^{2} \Gamma_{n, \epsilon}^{\frac{q-2+\alpha}{2}} \mathrm{~d} x .
\end{gathered}
$$

Then we use (1.3), (1.4) and (2.1) to obtain

$$
\begin{aligned}
J & \leq c \int_{B} \eta^{2} D^{2} F\left(\nabla u_{\epsilon}\right)\left(\partial_{n} \nabla u_{\epsilon}, \partial_{n} \nabla u_{\epsilon}\right) \Gamma_{n, \epsilon}^{\frac{\alpha}{2}} \mathrm{~d} x \\
& \leq c \int_{B} D^{2} F\left(\nabla u_{\epsilon}\right)(\nabla \eta, \nabla \eta) \Gamma_{n, \epsilon}^{\frac{\alpha+2}{2}} \mathrm{~d} x \\
& \leq c\left[\int_{B} \tilde{\Gamma}_{\epsilon}^{\frac{p-2}{2}} \Gamma_{n, \epsilon}^{\frac{\alpha+2}{2}}|\nabla \eta|^{2} \mathrm{~d} x+\int_{B}|\nabla \eta|^{2} \Gamma_{n, \epsilon}^{\frac{q+\alpha}{2}} \mathrm{~d} x\right] .
\end{aligned}
$$

Inserting this into (2.5) it follows

$$
\int_{B} \eta^{2} \Gamma_{n, \epsilon}^{\frac{q+2+\alpha}{2}} \mathrm{~d} x \leq c\left[\int_{B}\left(\eta^{2}+|\nabla \eta|^{2}\right) \Gamma_{n, \epsilon}^{\frac{q+\alpha}{2}} \mathrm{~d} x+\int_{B} \tilde{\Gamma}_{\epsilon}^{\frac{p-2}{2}} \Gamma_{n, \epsilon}^{\frac{\alpha+2}{2}}|\nabla \eta|^{2} \mathrm{~d} x\right] .
$$

Next we let $\beta \geq 0$ and fix $\eta$ as above. Then (recall that the sum is taken w.r.t. $\gamma=1, \ldots, n-1)$ we consider the expression

$$
\int_{B} \eta^{2} \tilde{\Gamma}_{\epsilon}^{\frac{p+2+\beta}{2}} \mathrm{~d} x
$$


perform an integration by parts in

$$
\int_{B} \partial_{\gamma} u_{\epsilon} \partial_{\gamma} u_{\epsilon} \eta^{2} \tilde{\Gamma}_{\epsilon}^{\frac{p+\beta}{2}} \mathrm{~d} x
$$

and carry out analogous calculations leading to the following variant of (2.5):

$$
\int_{B} \eta^{2} \tilde{\Gamma}_{\epsilon}^{\frac{p+2+\beta}{2}} \mathrm{~d} x \leq c\left[\int_{B}\left(\eta^{2}+|\nabla \eta|^{2}\right) \tilde{\Gamma}_{\epsilon}^{\frac{p+\beta}{2}} \mathrm{~d} x+\int_{B} \eta^{2}\left|\tilde{\nabla}^{2} u_{\epsilon}\right|^{2} \tilde{\Gamma}_{\epsilon}^{\frac{p-2+\beta}{2}} \mathrm{~d} x\right] .
$$

The second item on the r.h.s. of (2.7) is handled in the same manner as $J$ from above, i.e. this expression is bounded by

$$
\begin{aligned}
& c \int_{B} \eta^{2} D^{2} F\left(\nabla u_{\epsilon}\right)\left(\partial_{\gamma} \nabla u_{\epsilon}, \partial_{\gamma} \nabla u_{\epsilon}\right) \tilde{\Gamma}_{\epsilon}^{\frac{\beta}{2}} \mathrm{~d} x \\
& \quad \leq c\left[\int_{B}|\nabla \eta|^{2} \tilde{\Gamma}_{\epsilon}^{\frac{p+\beta}{2}} \mathrm{~d} x+\int_{B}|\nabla \eta|^{2} \Gamma_{n, \epsilon}^{\frac{q-2}{2}} \tilde{\Gamma}_{\epsilon}^{\frac{\beta+2}{2}} \mathrm{~d} x\right],
\end{aligned}
$$

thus we get

$$
\int_{B} \eta^{2} \tilde{\Gamma}_{\epsilon}^{\frac{p+2+\beta}{2}} \mathrm{~d} x \leq c\left[\int_{B}\left(\eta^{2}+|\nabla \eta|^{2}\right) \tilde{\Gamma}_{\epsilon}^{\frac{p+\beta}{2}} \mathrm{~d} x+\int_{B}|\nabla \eta|^{2} \Gamma_{n, \epsilon}^{\frac{q-2}{2}} \tilde{\Gamma}_{\epsilon}^{\frac{\beta+2}{2}} \mathrm{~d} x\right] .
$$

We return to (2.6) replacing $\eta$ by $\eta^{k}$ for a large number $k \in \mathbb{N}$, apply Young's inequality on the r.h.s. in order to get terms of the form $\tau \int_{B} \eta^{2 k} \Gamma_{n, \epsilon^{\frac{q+2+\alpha}{2}}}^{\mathrm{d} x}$ which can be absorbed in the 1.h.s. and get

$$
\begin{aligned}
\int_{B} \eta^{2 k} \Gamma_{n, \epsilon^{2}}^{\frac{q+2+\alpha}{2}} \mathrm{~d} x \leq & c\left[\int_{B}\left(\eta^{2 k}+|\nabla \eta|^{q+2+\alpha} \eta^{2 k-(q+2+\alpha)}\right) \mathrm{d} x\right. \\
& \left.+\int_{B}|\nabla \eta|^{\frac{2}{q}(\alpha+q+2)} \eta^{2 k-\frac{2}{q}(\alpha+q+2)} \tilde{\Gamma}_{\epsilon}^{\frac{p-2}{2} \frac{\alpha+q+2}{q}} \mathrm{~d} x\right] .
\end{aligned}
$$

In the same way (2.8) implies

$$
\begin{aligned}
\int_{B} \eta^{2 k} \tilde{\Gamma}_{\epsilon}^{\frac{p+2+\beta}{2}} \mathrm{~d} x \leq & c\left[\int_{B}\left(\eta^{2 k}+|\nabla \eta|^{p+2+\beta} \eta^{2 k-(p+2+\beta)}\right) \mathrm{d} x\right. \\
& \left.+\int_{B}|\nabla \eta|^{\frac{2}{p}(\beta+2+p)} \eta^{2 k-\frac{2}{p}(p+2+\beta)} \Gamma_{n, \epsilon}^{\frac{q-2}{2} \frac{p+2+\beta}{p}} \mathrm{~d} x\right] .
\end{aligned}
$$


Let us introduce the quantities

$$
\begin{aligned}
& a(\alpha, \rho):=\int_{B_{\rho}} \Gamma_{n, \epsilon}^{\frac{q+2+\alpha}{2}} \mathrm{~d} x, \\
& A(\alpha, \rho):=\int_{B_{\rho}} \tilde{\Gamma}_{\epsilon}^{\frac{p-2}{2} \frac{\alpha+q+2}{q}} \mathrm{~d} x, \\
& b(\beta, \rho):=\int_{B_{\rho}} \tilde{\Gamma}_{\epsilon}^{\frac{p+2+\beta}{2}} \mathrm{~d} x, \\
& B(\beta, \rho):=\int_{B_{\rho}} \Gamma_{n, \epsilon}^{\frac{q-2}{2} \frac{p+2+\beta}{p}} \mathrm{~d} x,
\end{aligned}
$$

where $B_{\rho}:=B_{\rho}\left(x_{0}\right)$ with $\rho<R$. If $p \leq 2$, then (2.9) immediately implies the uniform local integrability of $\partial_{n} u_{\epsilon}$ for any finite exponent $m$, and using this information in (2.10) we get the same result for $\tilde{\nabla} u_{\epsilon}$. So le us assume $p>2$. Then we have

$$
A\left(\alpha_{0}, \rho\right) \leq c(\rho)<\infty
$$

for a constant $c(\rho)$ going to infinity as $\rho \uparrow R$ but being independent of $\varepsilon$, provided we require

$$
\frac{p-2}{2} \frac{q+\alpha_{0}+2}{q} \leq \frac{p}{2}
$$

and quote Lemma $2.1 \mathrm{i})$. Note that on account of $(p-2)(q+2)<p q(2.12)$ holds for suitable positive numbers $\alpha_{0}$ and we may choose

$$
\alpha_{0}:=\frac{p q}{p-2}-(q+2)>0 .
$$

(2.11) combined with (2.9) shows that

$$
a\left(\alpha_{0}, \rho\right) \leq c(\rho)
$$

Now we select $\beta_{0}$ such that

$$
\frac{q-2}{2} \frac{p+\beta_{0}+2}{p}=\frac{q+2+\alpha_{0}}{2},
$$

hence $B\left(\beta_{0}, \rho\right) \leq c(\rho)$ on account of (2.14) and the definition of $B(\beta, \rho)$. Note that by the definition of $\alpha_{0}$ we have

$$
\frac{q-2}{2} \frac{p+2}{2}<\frac{q+\alpha_{0}+2}{2}
$$

which is equivalent to $-4 q-2 p^{2}+8<0$ (recall that we assume $p>2$ ), thus the solution $\beta_{0}$ of (2.15) is a positive number. Returning to (2.11) we have shown that

$$
A\left(\alpha_{l}, \rho\right)+B\left(\beta_{l}, \rho\right) \leq c_{l}(\rho)
$$


at least for $l=0$. Suppose that $l \geq 1$ and that $\left(2.16_{l-1}\right)$ is valid. We then like to prove $\left(2.16_{l}\right)$ for suitable exponents $\alpha_{l}, \beta_{l}$. First, $B\left(\beta_{l-1}, \rho\right) \leq c_{l-1}(\rho)$ together with (2.10) gives $b\left(\beta_{l-1}, \rho\right) \leq c(\rho)$ for a new constant (depending on $\ell$ ) so that

$$
A\left(\alpha_{l}, \rho\right) \leq c(\rho),
$$

provided

$$
\frac{p-2}{2} \frac{\alpha_{l}+q+2}{q} \leq \frac{p+2+\beta_{l-1}}{2},
$$

and we may define

$$
\begin{aligned}
\alpha_{l} & =\frac{q}{p-2}\left(\beta_{l-1}+p+2\right)-(q+2) \\
& =\frac{q}{p-2} \beta_{l-1}+\frac{q(p+2)}{p-2}-(q+2) \\
& =\frac{q}{p-2} \beta_{l-1}+\frac{4 q-2 p+4}{p-2}
\end{aligned}
$$

to ensure (2.17). (2.17) together with (2.9) implies $a\left(\alpha_{l}, \rho\right) \leq c(\rho)$ so that

$$
B\left(\beta_{l}, \rho\right) \leq c(\rho),
$$

if $\beta_{l}$ satisfies

$$
\frac{q-2}{2} \frac{p+2+\beta_{l}}{p}=\frac{q+2+\alpha_{l}}{2},
$$

which means

$$
\beta_{l}=\frac{p}{q-2} \alpha_{l}+\frac{1}{q-2}[4 p+4-2 q] .
$$

Inserting (2.18) in (2.19) we see

$$
\begin{aligned}
\beta_{l} & =\frac{p q}{(p-2)(q-2)} \beta_{l-1}+\xi \\
\xi & :=\frac{1}{q-2}[4 p+4-2 q]+\frac{1}{q-2} \frac{1}{p-2} p[4 q-2 p+4] \\
& =\frac{2 p^{2}+2 p q+4 q-8}{(q-2)(p-2)}>0,
\end{aligned}
$$

hence the sequence $\beta_{l}$ consists of strictly positive numbers. Altogether we have shown the validity of $\left(2.16_{l}\right)$ for any $l$ provided $\left\{\alpha_{l}\right\},\left\{\beta_{l}\right\}$ are defined according to (2.18), (2.20) with the initial values from (2.13) and (2.15). Moreover, since

$$
\frac{p q}{(p-2)(q-2)}>1
$$


we see from (2.20) that $\beta_{l} \rightarrow \infty$ as $l \rightarrow \infty$, and (2.18) gives the same for $\alpha_{l}$ which means that

$$
\int_{B_{\rho}}\left|\nabla u_{\epsilon}\right|^{m} \mathrm{~d} x \leq c(m, \rho)
$$

for any $\rho<R$ and all $m<\infty$, the constant being independent of $\epsilon$. Now the claim of Theorem 1.1, i) follows from (2.21) combined with Lemma 2.1, i).

\section{Some auxiliary results}

In this section we will establish a regularity result which might be known but which we could not trace in the literature. As already remarked it will enable us to deduce part ii) of Theorem 1.1 from part i).

Lemma 3.1. Let $2 \leq s \leq t<\infty$ and suppose that our integrand functions $f$ : $\mathbb{R}^{n-1} \rightarrow[0, \infty), g: \mathbb{R} \rightarrow[0, \infty)$ satisfy (1.3) and (1.4) with exponents $s$ and $t$ respectively. Suppose also that $u \in W_{s, \mathrm{loc}}^{1}(\Omega)$ locally minimizes the functional $I[\cdot, \Omega]$. Then, if $\nabla u \in L_{\mathrm{loc}}^{\rho}\left(\Omega, \mathbb{R}^{n}\right)$ for any $\rho<\infty$, we have that $u \in C^{1, \mu}(\Omega)$ for all $\mu<1$.

Proof of Lemma 3.1. It is enough to show that $\nabla u$ is locally bounded. Then, passing to difference quotients, it is easy to see that $\Delta_{h} u$ is a solution of a uniformly elliptic equation with bounded measurable coefficients, all bounds being independent of $h$. The De Giorgi-Moser-Nash theory (see [13]) implies $\Delta_{h} u \in C^{0, \tilde{\mu}}(\Omega)$ uniformly in $h$ for some exponent $\tilde{\mu}>0$ so that $u \in C^{1, \tilde{\mu}}(\Omega)$. Then we differentiate the Euler equation (via difference quotients) and get for $v:=\partial_{\gamma} u, \gamma=1, \ldots$, $n$ the validity of

$$
\int_{\Omega} A(x)(\nabla v, \nabla \varphi) \mathrm{d} x=0 \quad \text { for every } \varphi \in C_{0}^{\infty}(\Omega),
$$

where $A(x)$ is the parameter dependent bilinear form $D^{2} F(\nabla u(x))$. Since $\nabla u$ is Hölder continuous with exponent $\tilde{\mu}$, we have the continuity and local uniform ellipticity of $A(x)$. From this $u \in C^{1, \mu}(\Omega)$ for all $\mu<1$ follows with the help of a standard pertubation argument, compare, e.g., [11].

We first use our assumption

$$
\nabla u \in L_{\mathrm{loc}}^{\rho}\left(\Omega ; \mathbb{R}^{n}\right) \quad \text { for all } \rho<\infty
$$

to show that $\nabla u$ is weakly differentiable. Let $e_{\gamma}$ denote a coordinate direction, $\gamma=1, \ldots, n$, and let

$$
\Delta_{h}^{\gamma} \Psi(x):=\frac{1}{h}\left(\Psi\left(x+h e_{\gamma}\right)-\Psi(x)\right), \quad h \neq 0
$$


denote the corresponding difference quotient of a function $\Psi$. The minimality of $u$ implies the Euler-equation $\left(\Omega^{\prime} \Subset \Omega\right)$

$$
\int_{\Omega^{\prime}} D F(\nabla u) \cdot \nabla \varphi \mathrm{d} x=0
$$

valid for $\varphi \in C_{0}^{\infty}\left(\Omega^{\prime}\right)$ but according to (3.1) $\varphi$ can be taken from any space $\stackrel{\circ}{W}_{\tau}^{1}\left(\Omega^{\prime}\right)$, $\tau>1$, in particular we may choose $\varphi=\Delta_{-h}^{\gamma}\left(\eta^{2} \Delta_{h}^{\gamma} u\right)$, where $\eta \in C_{0}^{\infty}(\Omega)$ is fixed and $|h| \ll 1$ is sufficiently small. From (3.2) we get

$$
\int_{\Omega} \Delta_{h}^{\gamma}(D F(\nabla u)) \cdot \nabla\left(\eta^{2} \Delta_{h}^{\gamma} u\right) \mathrm{d} x=0,
$$

and if we introduce the bilinear form

$$
\mathcal{B}_{h}^{\gamma}:=\int_{0}^{1} D^{2} F\left(\nabla u+t h \Delta_{h}^{\gamma} \nabla u\right) \mathrm{d} t,
$$

then (3.3) takes the form (no summation w.r.t. $\gamma$ )

$$
\int_{\Omega} \mathcal{B}_{h}^{\gamma}\left(\Delta_{h}^{\gamma} \nabla u, \Delta_{h}^{\gamma} \nabla u\right) \eta^{2} \mathrm{~d} x=-2 \int_{\Omega} \eta \mathcal{B}\left(\Delta_{h}^{\gamma} \nabla u, \nabla \eta\right) \Delta_{h}^{\gamma} u \mathrm{~d} x .
$$

Using on the r.h.s. the Cauchy-Schwarz inequality for the bilinear form $\mathcal{B}$ together with Young's inequality we deduce from (3.4) the estimate

$$
\int_{\Omega} \mathcal{B}_{h}^{\gamma}\left(\Delta_{h}^{\gamma} \nabla u, \Delta_{h}^{\gamma} \nabla u\right) \eta^{2} \mathrm{~d} x \leq c \int_{\Omega} \mathcal{B}_{h}^{\gamma}(\nabla \eta, \nabla \eta)\left|\Delta_{h}^{\gamma} u\right|^{2} \mathrm{~d} x .
$$

Elementary properties of the difference quotients in combination with (3.1) show that

$$
\int_{\Omega} \mathcal{B}_{h}^{\gamma}(\nabla \eta, \nabla \eta)\left|\Delta_{h}^{\gamma} u\right|^{2} \mathrm{~d} x \longrightarrow \int_{\Omega} D^{2} F(\nabla u)(\nabla \eta, \nabla \eta)\left|\partial_{\gamma} u\right|^{2} \mathrm{~d} x
$$

as $h \rightarrow 0$, the limit of course being finite on acount of (3.1). Since $s \geq 2$ the 1.h.s. of (3.5) is bounded from below by $c \int_{\Omega} \eta^{2}\left|\Delta_{h}^{\gamma} \nabla u\right|^{2} \mathrm{~d} x$, hence

$$
\int_{\Omega} \eta^{2}\left|\Delta_{h}^{\gamma} \nabla u\right|^{2} \mathrm{~d} x \leq c(\eta)<\infty
$$

for $|h| \ll 1$, and since (3.6) holds for any direction and arbitrary $\eta \in C_{0}^{\infty}(\Omega)$, it follows that $u$ is in the space $W_{2, \text { loc }}^{2}(\Omega)$, in particular it holds $\Delta_{h}^{\gamma} \nabla u \longrightarrow \partial_{\gamma} \nabla u$ a.e. Since $\mathcal{B}_{h}^{\gamma}\left(\Delta_{h}^{\gamma} \nabla u, \Delta_{h}^{\gamma} \nabla u\right) \geq 0$ we may therefore apply Fatou's lemma on the 1.h.s. of (3.5) leading to the inequality

$$
\int_{\Omega} \eta^{2} D^{2} F(\nabla u)\left(\partial_{\gamma} \nabla u, \partial_{\gamma} \nabla u\right) \mathrm{d} x \leq c \int_{\Omega} D^{2} F(\nabla u)(\nabla \eta, \nabla \eta)\left|\partial_{\gamma} u\right|^{2} \mathrm{~d} x .
$$


Note that (3.7) can also be established if $s<2$, we refer to [2], page 56. Now we fix a ball $B:=B_{R}\left(x_{0}\right)$ with compact closure in $\Omega$. Let $0<r<\hat{r}<R$ (all balls are centered at $\left.x_{0}\right), k>0$, and define

$$
\begin{aligned}
& \Gamma_{n}:=1+\left|\partial_{n} u\right|^{2}, \Gamma_{n}^{(h)}:=1+\left|\Delta_{h}^{n} u\right|^{2}, \\
& \widetilde{\Gamma}:=1+|\tilde{\nabla} u|^{2}, \quad \tilde{\nabla} u:=\left(\partial_{1} u, \ldots, \partial_{n-1} u\right) .
\end{aligned}
$$

Then, choosing $\eta \in C_{0}^{\infty}\left(B_{\hat{r}}\right), \varphi:=\Delta_{-h}^{n}\left(\eta^{2} \Delta_{h}^{n} u\left[\Gamma_{n}^{(h)}-k\right]^{+}\right)$is admissible in (3.2) leading to

$$
\begin{aligned}
0= & \int_{B_{\hat{r}}} \mathcal{B}_{h}^{n}\left(\nabla \Delta_{h}^{n} u, \nabla\left(\eta^{2} \Delta_{h}^{n} u\left[\Gamma_{n}^{(h)}-k\right]^{+}\right)\right) \mathrm{d} x \\
= & \int_{B_{\hat{r}}} \eta^{2}\left[\Gamma_{n}^{(h)}-k\right]^{+} \mathcal{B}_{h}^{n}\left(\nabla \Delta_{h}^{n} u, \nabla \Delta_{h}^{n} u\right) \mathrm{d} x \\
& +\int_{B_{\hat{r}} \cap\left[\Gamma_{n}^{(h)} \geq k\right]} \eta^{2} \mathcal{B}_{h}^{n}\left(\nabla \Delta_{h}^{n} u, \nabla \Gamma_{n}^{(h)}\right) \Delta_{h}^{n} u \mathrm{~d} x \\
& +\int_{B_{\hat{r}}} 2 \eta \mathcal{B}_{h}^{n}\left(\nabla \Delta_{h}^{n} u, \nabla \eta\right)\left[\Gamma_{n}^{(h)}-k\right]^{+} \Delta_{h}^{n} u \mathrm{~d} x \\
= & : T_{1}+T_{2}+T_{3} .
\end{aligned}
$$

$T_{1}$ is non-negative, and clearly

$$
\begin{aligned}
& T_{2}=\frac{1}{2} \int_{B_{\hat{r}} \cap\left[\Gamma_{n}^{(h)} \geq k\right]} \eta^{2} \mathcal{B}_{h}^{n}\left(\nabla \Gamma_{n}^{(h)}, \nabla \Gamma_{n}^{(h)}\right) \mathrm{d} x, \\
& T_{3}=\int_{B_{\hat{r}} \cap\left[\Gamma_{n}^{(h)} \geq k\right]} \eta \mathcal{B}_{h}^{n}\left(\nabla \Gamma_{n}^{(h)}, \nabla \eta\right)\left(\Gamma_{n}^{(h)}-k\right) \mathrm{d} x,
\end{aligned}
$$

hence

$$
\begin{aligned}
& \int_{B_{\hat{r}} \cap\left[\Gamma_{n}^{(h)} \geq k\right]} \eta^{2} \mathcal{B}_{h}^{n}\left(\nabla \Gamma_{n}^{(h)}, \nabla \Gamma_{n}^{(h)}\right) \mathrm{d} x \\
& \quad \leq-2 \int_{B_{\hat{r}} \cap\left[\Gamma_{n}^{(h)} \geq k\right]} \eta \mathcal{B}_{h}^{n}\left(\nabla \Gamma_{n}^{(h)}, \nabla \eta\right)\left(\Gamma_{n}^{(h)}-k\right) \mathrm{d} x .
\end{aligned}
$$

The same arguments leading from (3.4) to (3.5) then show

$$
\begin{aligned}
& \int_{B_{\hat{r}} \cap\left[\Gamma_{n}^{(h)} \geq k\right]} \eta^{2} \mathcal{B}_{h}^{n}\left(\nabla \Gamma_{n}^{(h)}, \nabla \Gamma_{n}^{(h)}\right) \mathrm{d} x \\
& \quad \leq c \int_{B_{\hat{r}} \cap\left[\Gamma_{n}^{(h)} \geq k\right]} \mathcal{B}_{h}^{n}(\nabla \eta, \nabla \eta)\left(\Gamma_{n}^{(h)}-k\right)^{2} \mathrm{~d} x .
\end{aligned}
$$


Again by (3.1) it is immediate that

$$
\text { r.h.s. of (3.8) } \underset{h \rightarrow 0}{\longrightarrow} c \int_{B_{\hat{r}} \cap\left[\Gamma_{n} \geq k\right]} D^{2} F(\nabla u)(\nabla \eta, \nabla \eta)\left(\Gamma_{n}-k\right)^{2} \mathrm{~d} x,
$$

whereas on the 1.h.s. of (3.8) we observe that the integrand is $\geq 0$ and pointwise convergent ( since $u \in W_{2, \text { loc }}^{2}(\Omega)$ ), thus Fatou's lemma is applicable, and we deduce from (3.8)

$$
\begin{aligned}
& \int_{B_{\hat{r}} \cap\left[\Gamma_{n} \geq k\right]} \eta^{2} D^{2} F(\nabla u)\left(\nabla \Gamma_{n}, \nabla \Gamma_{n}\right) \mathrm{d} x \\
& \quad \leq c \int_{B_{\hat{r}} \cap\left[\Gamma_{n} \geq k\right]} D^{2} F(\nabla u)(\nabla \eta, \nabla \eta)\left(\Gamma_{n}-k\right)^{2} \mathrm{~d} x .
\end{aligned}
$$

Recalling the ellipticity estimates for $f$ and $g$ we get from (3.9) the Caccioppolitype inequality

$$
\begin{aligned}
\int_{B_{\hat{r}} \cap\left[\Gamma_{n} \geq k\right]} \eta^{2}\left|\nabla \Gamma_{n}\right|^{2} \mathrm{~d} x \leq & c\left[\int_{B_{\hat{r}} \cap\left[\Gamma_{n} \geq k\right]} \tilde{\Gamma}^{\frac{s-2}{2}}\left(\Gamma_{n}-k\right)^{2}|\nabla \eta|^{2} \mathrm{~d} x\right. \\
& \left.+\int_{B_{\hat{r}} \cap\left[\Gamma_{n} \geq k\right]} \Gamma_{n}^{\frac{t-2}{2}}\left(\Gamma_{n}-k\right)^{2}|\nabla \eta|^{2} \mathrm{~d} x\right] .
\end{aligned}
$$

Let $\eta=1$ on $B_{r},|\nabla \eta| \leq c /(\hat{r}-r), 0 \leq \eta \leq 1$. Let us further abbreviate

$$
A(k, r):=B_{r} \cap\left[\Gamma_{n} \geq k\right] .
$$

We proceed similar to [2], proof of Theorem 5.22: we have

$$
\begin{aligned}
& \int_{A(k, r)}\left(\Gamma_{n}-k\right)^{\frac{n}{n-1}} \mathrm{~d} x \leq \int_{B_{\hat{r}}}\left[\eta\left(\Gamma_{n}-k\right)^{+}\right]^{\frac{n}{n-1}} \mathrm{~d} x \\
& \leq c\left[\int_{B_{\hat{r}}}\left|\nabla\left(\eta\left(\Gamma_{n}-k\right)^{+}\right)\right| \mathrm{d} x\right]^{\frac{n}{n-1}} \\
& \leq c\left[I_{1}^{\frac{n}{n-1}}+I_{2}^{\frac{n}{n-1}}\right], \\
& I_{1}^{\frac{n}{n-1}}:=\left[\int_{A(k, r)}|\nabla \eta|\left(\Gamma_{n}-k\right) \mathrm{d} x\right]^{\frac{n}{n-1}} \\
& \leq c(\hat{r}-r)^{-\frac{n}{n-1}}\left[\int_{A(k, \hat{r})} \Gamma_{n}^{\frac{t-2}{4}}\left(\Gamma_{n}-k\right) \Gamma_{n}^{\frac{2-t}{4}}\right]^{\frac{n}{n-1}} \\
& \leq c(\hat{r}-r)^{-\frac{n}{n-1}}\left[\int_{A(k, \hat{r})} \Gamma_{n}^{\frac{t-2}{2}}\left(\Gamma_{n}-k\right)^{2} \mathrm{~d} x\right]^{\frac{1}{2} \frac{n}{n-1}}\left[\int_{A(k, \hat{r})} \Gamma_{n}^{\frac{2-t}{2}} \mathrm{~d} x\right]^{\frac{1}{2} \frac{n}{n-1}},
\end{aligned}
$$




$$
\begin{aligned}
I_{2}^{\frac{n}{n-1}}: & =\left[\int_{A(k, \hat{r})} \eta\left|\nabla \Gamma_{n}\right| \mathrm{d} x\right]^{\frac{n}{n-1}} \\
& \leq c\left[\int_{A(k, \hat{r})} \eta^{2}\left|\nabla \Gamma_{n}\right|^{2} \mathrm{~d} x\right]^{\frac{1}{2} \frac{n}{n-1}}|A(k, \hat{r})|^{\frac{1}{2} \frac{n}{n-1}},
\end{aligned}
$$

where in (3.11) we made use of Sobolev's inequality, whereas (3.12) and (3.13) just follow from Hölder's inequality. Inserting (3.10) into the r.h.s. of (3.13), we get

$$
\begin{aligned}
& \int_{A(k, r)}\left(\Gamma_{n}-k\right)^{\frac{n}{n-1}} \mathrm{~d} x \\
& \leq c(\hat{r}-r)^{-\frac{n}{n-1}}\left\{\left[\int_{A(k, \hat{r})} \Gamma_{n}^{\frac{t-2}{2}}\left(\Gamma_{n}-k\right)^{2} \mathrm{~d} x\right]^{\frac{1}{2} \frac{n}{n-1}}\left[\int_{A(k, \hat{r})} \Gamma_{n}^{\frac{2-t}{2}} \mathrm{~d} x\right]^{\frac{1}{2} \frac{n}{n-1}}\right. \\
& \left.+\left[\int_{A(k, \hat{r})} \Gamma_{n}^{\frac{t-2}{2}}\left(\Gamma_{n}-k\right)^{2} \mathrm{~d} x+\int_{A(k, \hat{r})} \tilde{\Gamma}^{\frac{s-2}{2}}\left(\Gamma_{n}-k\right)^{2} \mathrm{~d} x\right]^{\frac{1}{2} \frac{n}{n-1}}|A(k, \hat{r})|^{\frac{1}{2} \frac{n}{n-1}}\right\} .
\end{aligned}
$$

Let $v, \varkappa>1$ be arbitrary for the moment. We recall (3.1) and apply Hölder's inequality to obtain

$$
\begin{aligned}
\int_{A(k, r)} \Gamma_{n}^{\frac{t-2}{2}}\left(\Gamma_{n}-k\right)^{2} \mathrm{~d} x & \leq \int_{A(k, r)}\left(\Gamma_{n}-k\right)^{\frac{n}{n-1} \frac{1}{v}} \Gamma_{n}^{2-\frac{n}{n-1} \frac{1}{v}} \Gamma_{n}^{\frac{t-2}{2}} \mathrm{~d} x \\
& \leq c_{1}(v)\left[\int_{A(k, r)}\left(\Gamma_{n}-k\right)^{\frac{n}{n-1}} \mathrm{~d} x\right]^{\frac{1}{v}}, \\
\int_{A(k, r)} \tilde{\Gamma}^{\frac{s-2}{2}}\left(\Gamma_{n}-k\right)^{2} \mathrm{~d} x & =\int_{A(k, r)}\left(\Gamma_{n}-k\right)^{\frac{n}{n-1} \frac{1}{v}}\left(\Gamma_{n}-k\right)^{2-\frac{n}{n-1} \frac{1}{v}} \tilde{\Gamma}^{\frac{s-2}{2}} \mathrm{~d} x \\
& \leq \int_{A(k, r)}\left(\Gamma_{n}-k\right)^{\frac{n}{n-1} \frac{1}{v}} \Gamma_{n}^{2-\frac{n}{n-1} \frac{1}{v}} \tilde{\Gamma}^{\frac{s-2}{2}} \mathrm{~d} x \\
& \leq c_{1}(v)\left[\int_{A(k, r)}\left(\Gamma_{n}-k\right)^{\frac{n}{n-1}} \mathrm{~d} x\right]^{\frac{1}{v}},
\end{aligned}
$$


$c_{1}(v)$ denoting a local constant depending on our fixed ball $B_{R}\left(x_{0}\right)$. In the same way it follows

$$
\begin{gathered}
\int_{A(k, \hat{r})} \Gamma_{n}^{\frac{2-t}{2}} \mathrm{~d} x=\int_{A(k, \hat{r})} \Gamma_{n}^{\frac{1}{\varkappa} \frac{t-2}{2}} \Gamma_{n}^{\frac{2-t}{2}-\frac{1}{\varkappa} \frac{t-2}{2}} \mathrm{~d} x \leq c_{2}(\varkappa)\left[\int_{A(k, \hat{r})} \Gamma_{n}^{\frac{t-2}{2}} \mathrm{~d} x\right]^{\frac{1}{\varkappa}}, \\
|A(k, \hat{r})| \leq \int_{A(k, \hat{r})} \Gamma_{n}^{\frac{1}{\varkappa} \frac{t-2}{2}} \mathrm{~d} x \leq c_{2}(\varkappa)\left[\int_{A(k, \hat{r})} \Gamma_{n}^{\frac{t-2}{2}} \mathrm{~d} x\right]^{\frac{1}{\varkappa}} .
\end{gathered}
$$

This gives

$$
\begin{aligned}
\tau(k, r): & =\int_{A(k, r)}\left(\Gamma_{n}^{\frac{t-2}{2}}\left(\Gamma_{n}-k\right)^{2}+\tilde{\Gamma}^{\frac{s-2}{2}}\left(\Gamma_{n}-k\right)^{2}\right) \mathrm{d} x \\
& \leq c_{1}(\nu)\left[\int_{A(k, r)}\left(\Gamma_{n}-k\right)^{\frac{n}{n-1}} \mathrm{~d} x\right]^{\frac{1}{v}} \\
& \leq c_{3}(\nu, \varkappa)(\hat{r}-r)^{-\frac{1}{v} \frac{n}{n-1}} \tau(k, \hat{r})^{\frac{1}{v} \frac{1}{2} \frac{n}{n-1}}\left[\int_{A(k, \hat{r})} \Gamma_{n}^{\frac{t-2}{2}} \mathrm{~d} x\right]^{\frac{1}{\varkappa v} \frac{1}{2} \frac{n}{n-1}},
\end{aligned}
$$

where we made use of (3.15), (3.16) to obtain the first inequality, (3.14), (3.17) and (3.18) imply the second one. If we abbreviate

$$
a(k, r):=\int_{A(k, r)} \Gamma_{n}^{\frac{t-2}{2}} \mathrm{~d} x,
$$

then it is shown that for any $k>0$ and for all $v, \varkappa>1,0<r<\hat{r} \leq R$ the inequality

$$
\tau(k, r) \leq c_{3}(\hat{r}-r)^{-\frac{1}{v} \frac{n}{n-1}} \tau(k, \hat{r})^{\frac{1}{v} \frac{1}{2} \frac{n}{n-1}} a(k, \hat{r})^{\frac{1}{\varkappa v} \frac{1}{2} \frac{n}{n-1}}
$$

is valid. Obviously $a(h, \hat{r}) \leq(h-k)^{-2} \tau(k, \hat{r})$, if $h>k$, and from (3.19) (replacing $k$ by $h>k$ ) we get

$$
\begin{aligned}
\tau(h, r) & \leq c_{3}(\hat{r}-r)^{-\gamma} \tau(h, \hat{r})^{\frac{1}{v} \frac{1}{2} \frac{n}{n-1}} a(h, \hat{r})^{\frac{1}{\varkappa v} \frac{1}{2} \frac{n}{n-1}} \\
& \leq c_{3}(\hat{r}-r)^{-\gamma}(h-k)^{-\beta} \tau(h, \hat{r})^{\frac{1}{v} \frac{1}{2} \frac{n}{n-1}} \tau(k, \hat{r})^{\frac{1}{\varkappa v} \frac{1}{2} \frac{n}{n-1}}
\end{aligned}
$$

with suitable positive exponents $\gamma, \beta$. Since $\tau(h, \hat{r}) \leq \tau(k, \hat{r})$, we finally arrive at

$$
\begin{aligned}
\tau(h, r) & \leq c_{3}(\hat{r}-r)^{-\gamma}(h-k)^{-\beta} \tau(k, \hat{r})^{\frac{1}{2} \frac{n}{n-1} \frac{1}{\nu}\left[1+\frac{1}{\varkappa}\right]}, \\
0 & <k<h, 0<r<\hat{r} \leq R .
\end{aligned}
$$


In (3.20) $v$ and $\varkappa$ are still in our disposal, and for $v, \varkappa>1$ but very close to 1 we can arrange that

$$
\frac{1}{2} \frac{n}{n-1} \frac{1}{v}\left[1+\frac{1}{\varkappa}\right]>1 \text {, }
$$

thus a lemma of Stampacchia [18, Lemma 5.1, page 219] can be applied to (3.20) implying $\Gamma_{n} \in L^{\infty}\left(B_{R / 2}\left(x_{0}\right)\right)$. Since the ball $B_{R}\left(x_{0}\right)$ was arbitrary, it follows that $\partial_{n} u$ is in the space $L_{\mathrm{loc}}^{\infty}(\Omega)$.

Next we fix a coordinate direction $e_{m}, m<n$, and let $\Gamma_{m}:=1+\left|\partial_{m} u\right|^{2}$, $A(k, r):=B_{r} \cap\left[\Gamma_{m} \geq k\right]$ etc. We indicate the changes in the foregoing calculations: (3.9) holds for $\Gamma_{m}$ in place of $\Gamma_{n}$, and if we use $\partial_{n} u \in L_{\mathrm{loc}}^{\infty}(\Omega)$, then (3.10) can be replaced by

$$
\int_{A(k, \hat{r})} \eta^{2}\left|\nabla \Gamma_{m}\right|^{2} \mathrm{~d} x \leq c \int_{A(k, \hat{r})} \tilde{\Gamma}^{\frac{s-2}{2}}\left(\Gamma_{m}-k\right)^{2}|\nabla \eta|^{2} \mathrm{~d} x .
$$

Here we just observed $\Gamma_{n}^{\frac{t-2}{2}} \leq c \tilde{\Gamma}^{\frac{s-2}{2}}$. (3.11) remains valid for $\Gamma_{m}$, and we have

$$
I_{1}^{\frac{n}{n-1}} \leq c(\hat{r}-r)^{-\frac{n}{n-1}}\left[\int_{A(k, \hat{r})} \Gamma_{m}^{\frac{s-2}{2}}\left(\Gamma_{m}-k\right)^{2} \mathrm{~d} x\right]^{\frac{1}{2} \frac{n}{n-1}}\left[\int_{A(k, \hat{r})} \Gamma_{m}^{\frac{2-s}{2}} \mathrm{~d} x\right]^{\frac{1}{2} \frac{n}{n-1}} .
$$

(3.13) of course is true for $\Gamma_{m}$, and as before we can use $\left(3.10^{\prime}\right)$ on the r.h.s. of the $\Gamma_{m}$-version of (3.13), which leads in combination with $\left(3.12^{\prime}\right)$ and (3.13) to

$$
\begin{aligned}
& \int_{A(k, r)}\left(\Gamma_{m}-k\right)^{\frac{n}{n-1}} \mathrm{~d} x \\
& \leq c(\hat{r}-r)^{-\frac{n}{n-1}}\left\{\left[\int_{A(k, \hat{r})} \Gamma_{m}^{\frac{s-2}{2}}\left(\Gamma_{m}-k\right)^{2} \mathrm{~d} x\right]^{\frac{1}{2} \frac{n}{n-1}}\left[\int_{A(k, \hat{r})} \Gamma_{m}^{\frac{2-s}{2}} \mathrm{~d} x\right]^{\frac{1}{2} \frac{n}{n-1}}\right. \\
& \left.+\left[\int_{A(k, \hat{r})} \tilde{\Gamma}^{\frac{s-2}{2}}\left(\Gamma_{m}-k\right)^{2} \mathrm{~d} x\right]^{\frac{1}{2} \frac{n}{n-1}}|A(k, \hat{r})|^{\frac{1}{2} \frac{n}{n-1}}\right\} .
\end{aligned}
$$

Obviously (see (3.15), (3.16)) it holds for $v>1$ (recall (3.1)!)

$$
\int_{A(k, r)} \tilde{\Gamma}^{\frac{s-2}{2}}\left(\Gamma_{m}-k\right)^{2} \mathrm{~d} x \leq c_{1}(v)\left[\int_{A(k, r)}\left(\Gamma_{m}-k\right)^{\frac{n}{n-1}} \mathrm{~d} x\right]^{\frac{1}{v}},
$$

and in accordance with (3.17), (3.18) we find

$$
\begin{array}{r}
\int_{A(k, \hat{r})} \Gamma_{m}^{\frac{2-s}{2}} \mathrm{~d} x \leq c_{2}(\varkappa)\left[\int_{A(k, \hat{r})} \Gamma_{m}^{\frac{s-2}{2}} \mathrm{~d} x\right]^{\frac{1}{\varkappa}}, \\
|A(k, \hat{r})| \leq c_{2}(\varkappa)\left[\int_{A(k, \hat{r})} \Gamma_{m}^{\frac{s-2}{2}} \mathrm{~d} x\right]^{\frac{1}{\varkappa}} .
\end{array}
$$


This gives by $\left(3.14^{\prime}\right),\left(3.16^{\prime}\right)$ and $\left(3.17^{\prime}\right),\left(3.18^{\prime}\right)$, respectively,

$$
\begin{aligned}
& \int_{A(k, r)} \tilde{\Gamma}^{\frac{s-2}{2}}\left(\Gamma_{m}-k\right)^{2} \mathrm{~d} x \\
& \leq c_{1}(v)(\hat{r}-r)^{-\frac{1}{v} \frac{n}{n-1}}\left[\int_{A(k, \hat{r})} \tilde{\Gamma}^{\frac{s-2}{2}}\left(\Gamma_{m}-k\right)^{2} \mathrm{~d} x\right]^{\frac{1}{2} \frac{1}{v} \frac{n}{n-1}} \\
& \quad \cdot\left\{\left[\int_{A(k, \hat{r})} \Gamma_{m}^{\frac{2-s}{2}} \mathrm{~d} x\right]^{\frac{1}{2} \frac{n}{n-1}}+|A(k, \hat{r})|^{\frac{1}{2} \frac{n}{n-1}}\right\}^{\frac{1}{v}} \\
& \leq c_{3}(v, \varkappa)(\hat{r}-r)^{-\frac{1}{v} \frac{n}{n-1}}\left[\int_{A(k, \hat{r})} \tilde{\Gamma}^{\frac{s-2}{2}}\left(\Gamma_{m}-k\right)^{2} \mathrm{~d} x\right]^{\frac{1}{2} \frac{1}{v} \frac{n}{n-1}}\left[\int_{A(k, \hat{r})} \Gamma_{m}^{\frac{s-2}{2}} \mathrm{~d} x\right]^{\frac{1}{2} \frac{1}{v} \frac{1}{\varkappa} \frac{n}{n-1}} .
\end{aligned}
$$

Let us now define

$$
\begin{aligned}
\tau^{\prime}(k, r) & :=\int_{A(k, r)} \tilde{\Gamma}^{\frac{s-2}{2}}\left(\Gamma_{m}-k\right)^{2} \mathrm{~d} x, \\
a^{\prime}(k, r) & :=\int_{A(k, r)} \Gamma_{m}^{\frac{s-2}{2}} \mathrm{~d} x .
\end{aligned}
$$

Then the foregoing inequality implies (3.19) for the quantities $\tau^{\prime}, a^{\prime}$, and since again $(h>k)$

$$
a^{\prime}(h, \hat{r}) \leq(h-k)^{-2} \tau^{\prime}(k, \hat{r})
$$

we deduce (3.20) for $\tau^{\prime}$ which means $\Gamma_{m} \in L^{\infty}\left(B_{R / 2}\left(x_{0}\right)\right)$, thus $\partial_{m} u \in L_{\mathrm{loc}}^{\infty}(\Omega)$.

Remark 3.2. The information $\partial_{n} u \in L_{\text {loc }}^{\infty}(\Omega)$ is not really needed for the proof of $\tilde{\nabla} u \in L_{\text {loc }}^{\infty}\left(\Omega, \mathbb{R}^{n-1}\right)$. If we keep the quantity

$$
\int_{A(k, \hat{r})} \Gamma_{n}^{\frac{t-2}{2}}\left(\Gamma_{m}-k\right)^{2} \mathrm{~d} x
$$

on the r.h.s. of $\left(3.10^{\prime}\right)$, then this item occurs on the r.h.s. of (3.14') (it has to be added to $\left.\int_{A(k, \hat{r})} \tilde{\Gamma}^{\frac{s-2}{2}}\left(\Gamma_{m}-k\right)^{2} \mathrm{~d} x\right)$. On the other hand we have (compare $\left(3.16^{\prime}\right)$ )

$$
\int_{A(k, r)} \Gamma_{n}^{\frac{t-2}{2}}\left(\Gamma_{m}-k\right)^{2} \mathrm{~d} x \leq c_{1}(v)\left[\int_{A(k, r)}\left(\Gamma_{m}-k\right)^{\frac{n}{n-1}} \mathrm{~d} x\right]^{1 / v},
$$


hence

$$
\begin{aligned}
\tau^{\prime \prime}(k, r):= & \int_{A(k, r)}\left[\tilde{\Gamma}^{\frac{s-2}{2}}+\Gamma_{n}^{\frac{t-2}{2}}\right]\left(\Gamma_{m}-k\right)^{2} \mathrm{~d} x \\
\leq & c_{1}(v)\left[\int_{A(k, r)}\left(\Gamma_{m}-k\right)^{\frac{n}{n-1}} \mathrm{~d} x\right]^{1 / v} \\
\leq & c_{1}(v)(\hat{r}-r)^{-\frac{1}{v} \frac{n}{n-1}} \tau^{\prime \prime}(k, \hat{r})^{\frac{1}{2} \frac{1}{v} \frac{n}{n-1}}\left\{\left[\int_{A(k, \hat{r})} \Gamma_{m}^{\frac{2-s}{2}} \mathrm{~d} x\right]^{\frac{1}{2} \frac{n}{n-1}}\right. \\
& \left.+|A(k, \hat{r})|^{\frac{1}{2} \frac{n}{n-1}}\right\}^{\frac{1}{v}}
\end{aligned}
$$

and the terms in $\{\ldots\}$ are treated via $\left(3.17^{\prime}\right),\left(3.18^{\prime}\right)$. This gives (3.19) for $\tau^{\prime \prime}$ and $a^{\prime}$, and from $a^{\prime}(h, \hat{r}) \leq(h-k)^{-2} \tau^{\prime \prime}(k, \hat{r}), h>k$, the $\tau^{\prime \prime}$-version of (3.20) follows which again gives $\partial_{m} u \in L_{\mathrm{loc}}^{\infty}(\Omega)$.

\section{References}

[1] R. A. Adams, "Sobolev Spaces", Academic Press, New York-San Francisco-London, 1975.

[2] M. Bildhauer, "Convex Variational Problems: Linear, Nearly Linear and Anisotropic Growth Conditions", Lecture Notes in Mathematics 1818, Springer, Berlin-Heidelberg-New York, 2003.

[3] M. BildhAUeR and M. FuCHS, Elliptic variational problems with nonstandard growth, International Mathematical Series 1, In: "Nonlinear problems in mathematical physics and related topics I, in honor of Prof. O.A. Ladyzhenskaya", T. Rozhkovskaya (ed.), Novosibirsk, Russia, March 2002 (in Russian), 49-62; Kluwer/Plenum Publishers, June 2002 (in English), 53-66.

[4] M. BILDHAUER and M. FUCHS, Higher integrability of the gradient for vectorial minimizers of decomposable variational integrals, Manuscripta Math. 123 (2007), 269-283.

[5] M. Bildhauer, M. FuChS and G. Mingione, A priori gradient bounds and local $C^{1, \alpha}$ estimates for (double) obstacle problems under nonstandard growth conditions, Z. Anal. Anwendungen 20 (2001), 959-985.

[6] M. BILDHAUER, M. FUCHS and X. ZHONG, Variational integrals with a wide range of anisotropy, Algebra i Analiz. 18 (2006), 46-71.

[7] H. J. CHOE, Interior behaviour of minimizers for certain functionals with nonstandard growth, Nonlinear Anal. 19.10 (1992), 933-945.

[8] L. Esposito, F. LeOnetTi and G. Mingione, Regularity for minimizers of functionals with p-q growth, Nonlinear Differential Equations Appl. 6 (1999), 133-148.

[9] L. Esposito, F. LEONETTI and G. MingIONE, Regularity results for minimizers of irregular integrals with ( $p, q)$-growth, Forum. Math. 14 (2002), 245-272.

[10] N. FUSCO and C. SBORDONE, Some remarks on the regularity of minima of anisotropic integrals, Comm. Partial Differential Equatins 18 (1993), 153-167.

[11] M. Giaquinta, "Multiple Integrals in the Calculus of Variations and Nonlinear Elliptic Systems", Ann. Math. Studies 105, Princeton University Press, Princeton, 1983.

[12] M. Giaquinta, Growth conditions and regularity, a counterexample, Manuscripta Math. 59 (1987), 245-248. 
[13] D. Gilbarg and N. S. Trudinger, "Elliptic Partial Differential Equations of Second Order", Grundlehren der math. Wiss. 224, second ed., revised third print., Springer, BerlinHeidelberg-New York, 1998.

[14] M. C. Hong, Some remarks on the minimizers of variational integrals with non standard growth conditions, Boll. Unione Mat. Ital. Sez. B Artic. Ric. Mat. (7) 6-A (1992), 91-101.

[15] P. MARCELLINI, Regularity of minimizers of integrals of the calculus of variations with non standard growth conditions, Arch. Ration. Mech. Anal. 105 (1989), 267-284.

[16] P. MARCELLINI, Everywhere regularity for a class of elliptic systems without growth conditions, Ann. Scuola Norm. Sup. Pisa Cl. Sci. 23 (1996), 1-25.

[17] U. MASSARI and M. MirANDA, "Minimal Surfaces of Codimension One", North-Holland Mathematics Studies 91, North-Holland, Amsterdam-New York-Oxford, 1983.

[18] G. StAMPACCHIA, Le problème de Dirichlet pour les équations elliptiques du second ordre à coefficients discontinues, Ann. Inst. Fourier (Grenoble) 15.1 (1965), 189-258.

[19] N. N. URAL'TSEVA and A. B. URDALETOVA, The boundedness of the gradients of generalized solutions of degenerate quasilinear nonuniformly elliptic equations, Vestn. Leningr. Univ. Mat. Mekh. Astron. 4 (1983), 50-56 (in Russian); English translation: Vestn. Leningr. Univ. Math. 16 (1984), 263-270.

Saarland University

Department of Mathematics

P.O. Box 151150

D-66041 Saarbrücken, Germany.

bibi@math.uni-sb.de

fuchs@math.uni-sb.de

University of Jyväskylä

Department of Mathematics

and Statistics

P.O. Box 35

FIN-40014

University of Jyväskylä, Finland zhong@maths.jyu.fi 